\title{
LORENTZ FORCE MEMS MAGNETOMETER
}

\author{
M.J. Thompson and D.A. Horsley*
}

Mechanical and Aerospace Engineering, University of California, Davis, California, USA

\begin{abstract}
This paper describes a MEMS Lorentz-force navigation magnetometer whose fabrication is fully compatible with existing foundry processes used to manufacture MEMS inertial sensors. The magnetometer is shown to have a Brownian-noise limit and corresponding angular resolution of $3.4 \mathrm{nT} / \mathrm{rt}-\mathrm{Hz}, 0.03$ degrees $/ \mathrm{rt}-$ $\mathrm{Hz}$ when operated at $25 \mathrm{mTorr}$ and $62 \mathrm{nT} / \mathrm{rt}-\mathrm{Hz}, 0.56$ degrees $/ \mathrm{rt}-\mathrm{Hz}$ when operated at ambient pressure. To achieve Brownian-limited performance at atmospheric pressure parametric amplification was performed which amplifies the Lorentz force $40 \%$ more than the Brownian noise force and increases the field sensitivity of the device up to 82.5 -fold.
\end{abstract}

\section{INTRODUCTION \\ Compass Magnetometers}

MEMS Lorentz force magnetometers are advantageous over other magnetometers because they are free from hysteresis, require no specialized materials and can be monolithically integrated with other MEMS inertial sensors. MEMS Z-axis Lorentz force magnetometers have achieved resolutions of $200 \mathrm{nT} / \sqrt{\mathrm{Hz}}$ using foundry fabrication [1]. The device presented here, shown in Fig. 1, was earlier shown when operated at atmospheric pressure, to have an electronic noise-limited resolution of $1 \mu \mathrm{T} / \sqrt{ } \mathrm{Hz}$ with a natural frequency $\left(f_{n}\right)$ of $8468 \mathrm{~Hz}$ and a quality factor $(Q)$ of 48.8 [2]. To improve the performance of this device parametric amplification is utilized to increase the field sensitivity and secondly the device is operated in a 25 mTorr vacuum, so that the device becomes Brownian noise limited.

\section{Parametric Amplification}

Parametric amplification increases the force to displacement transduction of the sensor by modulating the mechanical spring constant at twice the natural frequency. Previous work on parametrically amplified Lorentz force magnetometers showed how the Lorentz and Brownian noise forces are both amplified and the amplification acts like increasing the quality factor $(Q)$ of the MEMS resonator [3].

\section{DESIGN AND OPERATION \\ Fabrication}

The device, shown in Fig. 1, was fabricated using the MEMSCAP SOIMUMPS foundry process with a $10 \mu \mathrm{m}$ device layer. The device is a resonant force sensor, which detects the displacement resulting from Lorentz force on current-carrying flexures. The current is modulated at the device's natural frequency $\left(f_{n}\right)$ to maximize the force sensitivity, given by $Q / k$, where $k$ is the mechanical stiffness [2]. Differential capacitors convert the motion of the device to an electrical output through JFET buffers.

\section{Lorentz force}

The beams on the folded flexure springs are the Lorentz force actuators [2,3]. A Lorentz force is applied to the shuttle when the current-carrying beams of the flexures are in a magnetic field. Folded flexure beams are advantageous because they prevent stress caused from constrained expansion due to ohmic heating [2].

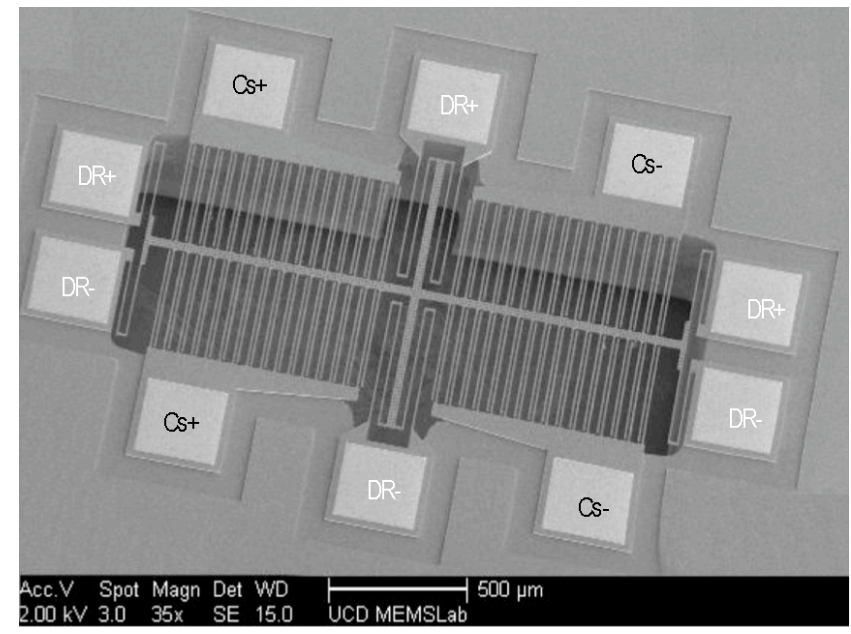

Figure 1. SEM of the magnetometer with three folded flexures used as Lorentz force actuators (Dr+, Dr-) and differential capacitive displacement detection (Cs+, $\mathrm{Cs}-$ ).

\section{Parametric Design}

Parametric amplification requires the mechanical spring constant to vary at twice the natural frequency and be phase locked with the excitation or drive input. In MEMS resonators, the nonlinear parallel plate capacitance is equivalent to a voltage controlled, negative spring stiffness [3]. To achieve parametric amplification, an AC voltage at twice the natural frequency $\left(2 f_{n}\right)$ is applied across the capacitors. For this device, the AC voltage $\left(f_{c}\right)$ is applied to the MEMS shuttle and DC voltage $\left(V_{b}\right)$ is applied to the other side of the capacitor through the JFET bias resistor.

\section{Electrical Circuit}

To create the current for the Lorentz force actuators, a voltage modulated at the natural frequency $\left(f_{n}\right)$ is applied to the top of the flexure $(D r+)$ and the inverse is applied to the bottom of the flexure $(D r-)$. This creates the required current but also creates a zero voltage node where the flexures join the shuttle. To control the voltage on the shuttle a signal is applied equally to the top and bottom of the flexure $\left(f_{c}\right)$. The motion is detected using displacement dependant differential parallel plate capacitors $(C s+$, $C s-)$. The output from the detection capacitors are buffered with high input impedance JFET's.

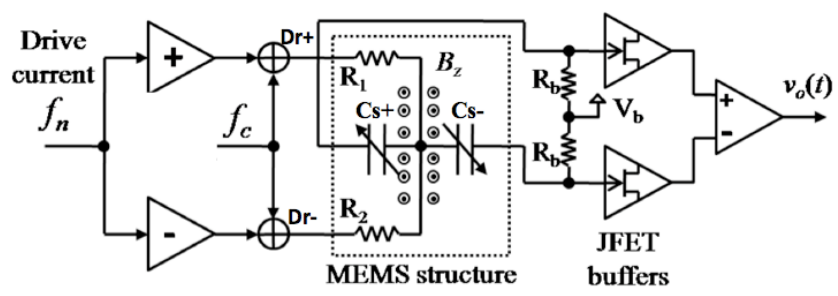

Figure 2. Electrical circuit for sensor drive and electronic detection. 
Parametric Amplification AC Magnetic Field

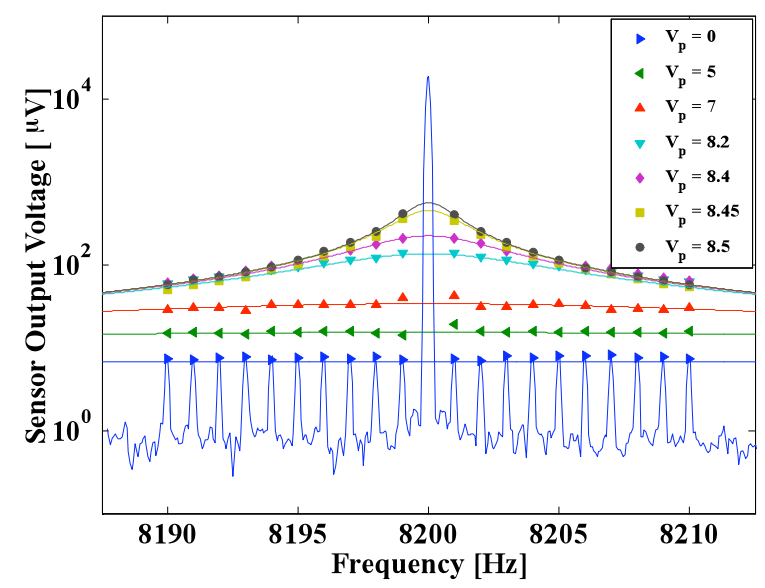

Figure 3. Sensor response at various pump voltages to $40 \mu \mathrm{T}$ magnetic field signals. The tone at $8.2 \mathrm{kHz}$ is capacitive feedthrough. With $V_{p}=0$, the sensitivity is constant over the $30 \mathrm{~Hz}$ measurement span, whereas at $V_{p}=8.5 \mathrm{~V}$ the $3 \mathrm{~dB}$ bandwidth is $\sim 1 \mathrm{~Hz}$.

\section{EXPERIMENTS}

Unless otherwise stated, the voltage bias is fixed at $18.6 \mathrm{~V}$ and the flexure current is $2.67 \mathrm{~mA}$. The device has $f_{n}=8200 \mathrm{~Hz}$ and $Q=48.8$ while operated in atmospheric conditions. The device was placed on top of an electromagnet. The electromagnet applied 10 test signals from $1 \mathrm{~Hz}$ to $10 \mathrm{~Hz}$ in $1 \mathrm{~Hz}$ increments with a $40 \mu \mathrm{T}$ amplitude.

\section{Parametric Amplification of the Lorentz Force}

The 10 test signals appear as sidebands around the drive frequency $(8.2 \mathrm{kHz})$ of the device as shown in Fig. 3. The full spectrum is shown for no parametric excitation. Capacitive feedthrough of the excitation signal at $f_{n}(8.2 \mathrm{kHz})$ corrupts the measurement at DC field but has no effect on the AC test signals. As the parametric pump is increased the figure tracks the response of the 10 test signals. To measure the increase in transduction, a second-order transfer function was fit to each frequency response by choosing an effective $Q_{\text {eff }}$ [3]. The increase in $Q$ is shown in Fig. 4 as a function of the applied parametric voltage and agrees well with the analytical model. At the highest pump voltage $\left(V_{p}=8.5 \mathrm{~V}\right)$, the sensitivity is increased 82.5 -fold, conferring an effective $Q_{\text {eff }}$ of 4026. As parametric amplification is increased the bandwidth of the device decreases with the original bandwidth of $85 \mathrm{~Hz}$ shrinking to $1 \mathrm{~Hz}$ for a parametric gain of 82.5 .

\section{Parametric Amplification of Brownian and Lorentz Forces}

The parametric voltage was set to give a parametric gain of 20 shown in Fig. 5. The full spectrum and a second-order data fit applied to the 10 signal peaks are shown. To measure the Brownian noise response the drive signal was turned off, so that the device was only excited by the parametric pump signal. The spectrum of the sensor output was averaged and a second-order fit applied. The fit obtained from the Lorentz force test tones was $40 \%$ greater (20) than the fit obtained from the Brownian noise force (14) for the same parametric pump excitation. This can be explained by a phenomenon called noise squeezing [4]. Noise squeezing occurs because parametric amplification is phase sensitive, with the in-phase signal being amplified and the inquadrature signal being attenuated. The Lorentz current drive is phase locked with the parametric signal to achieve maximum

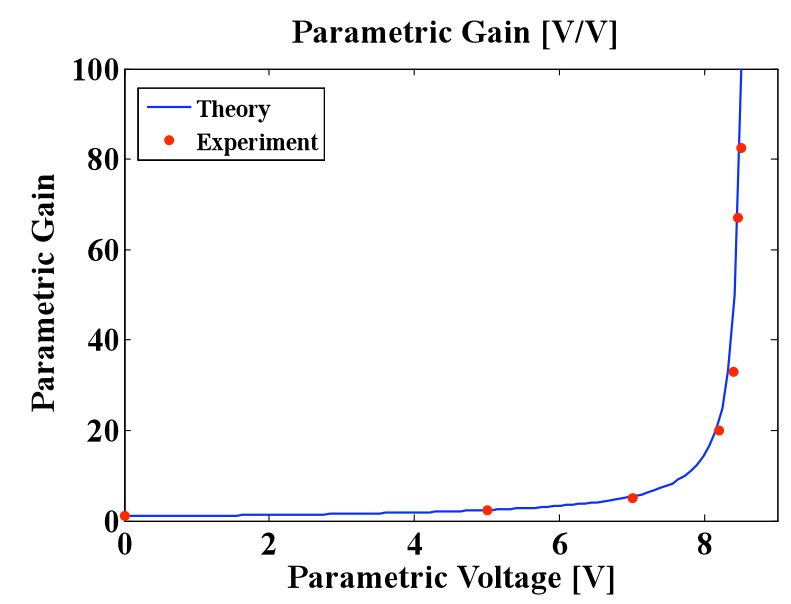

Figure 4. Parametric gain versus pump voltage amplitude.

amplification. However, the Brownian noise is white noise and spans over all phases; thus, the in-phase component is amplified and the in-quadrature component is attenuated. This fact leads to a reduction in the average noise amplification.

\section{Noise Squeezing}

The amplitude and phase time history of the sensor output is recorded in Fig. 5 with and without parametric amplification. When the device is not being parametrically amplified the in-phase and in-quadrature components of the noise are equal in amplitude and Johnson noise from the bias resistor $R_{b}$ dominates the response, shown in Fig. 2. When the device is parametrically excited, the output noise distribution becomes elliptical. The elliptical shape is caused by the phase sensitive amplification, noise squeezing, of the Brownian noise force. The in-phase component of the Brownian noise force is amplified while the out-of-phase component is attenuated. However, the attenuation of the out-of-phase component is not measured here because the Johnson noise dominates. Fig. 5 demonstrates how parametric amplification increases the force to displacement transduction so that the sensor can measure the Brownian noise force and becomes Brownian noise limited.

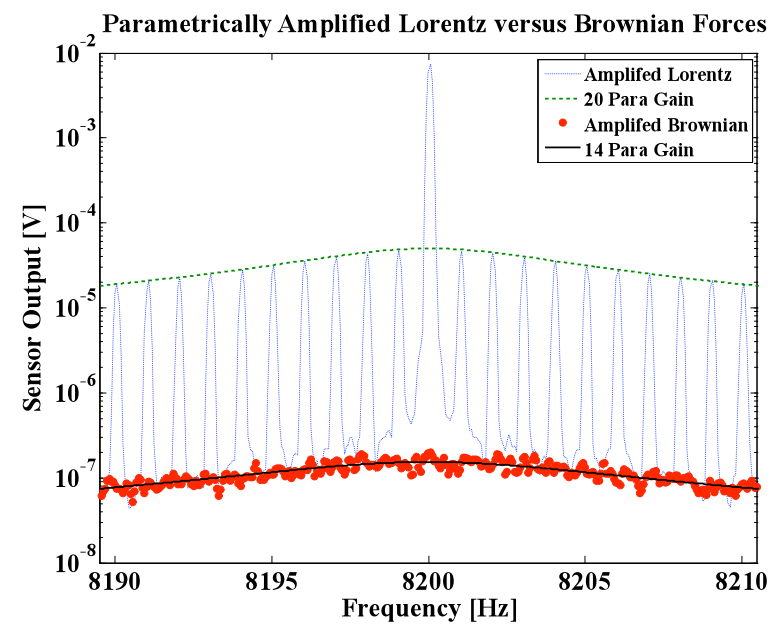

Figure 5. The spectrum and second-order fit for the device with the pump signal fixed at $8.3 \mathrm{~V}$ with the drive signal on, to measure the Lorentz force response, and with the drive off, to measure the Brownian noise response. 


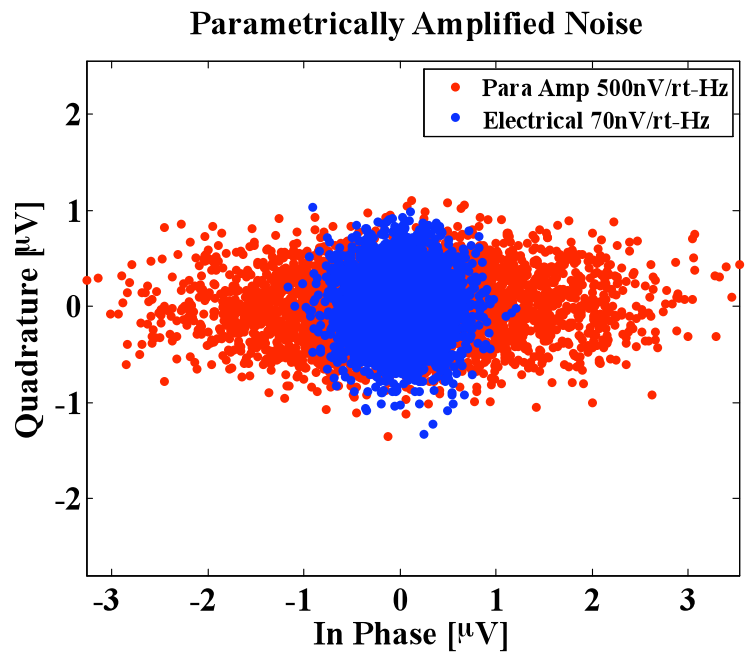

Figure 6. Time history of the amplitude and phase of the sensor output for no excitation and for an $8.4 \mathrm{~V}$ parametric excitation.

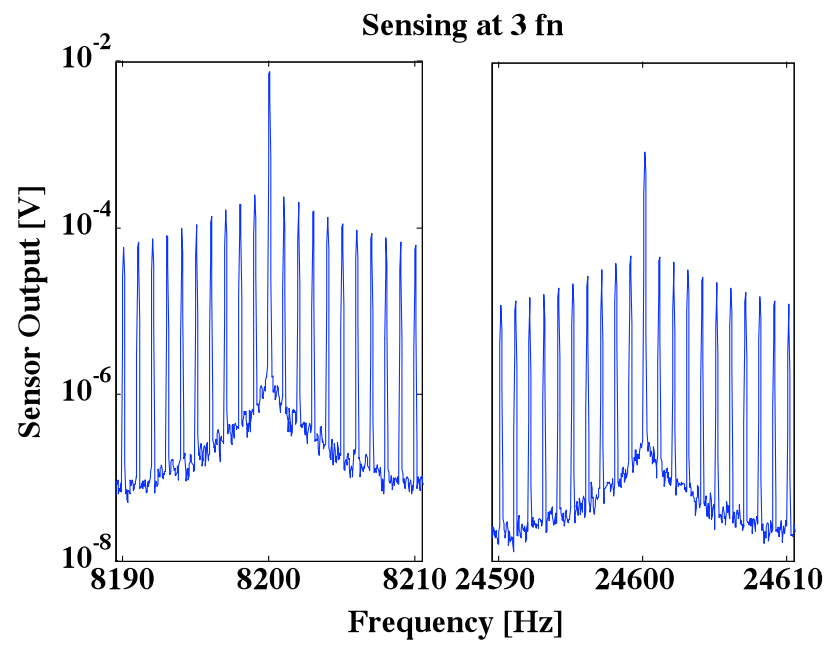

Figure 7. Sensor output at $f_{n}(8.2 \mathrm{kHz})$ and at $3 f_{n}(24.6 \mathrm{kHz})$.

\section{Sensing at $3 f_{n}$}

The detection of DC magnetic fields is corrupted by the drive to sense capacitive feedthrough, which results in the large peak at $8.2 \mathrm{kHz}$ in Fig.'s 3, 5 and 7. One method to bypass feedthrough is to drive and sense at different frequencies. The parametric pump signal applied to the MEMS shuttle causes the sense motion signal at $f_{n}$ to be mixed with the parametric signal at $2 f_{n}$. This mixing creates two extra signals, one at $f_{n}$ and one at $3 f_{n}$. The signal at $f_{n}$ is 90 degrees phase shifted from the sense signal at $f_{n}$ and adds $2.6 \%$ to the final amplitude and a 12 degree phase shift. The response of the magnetometer is shown at $f_{n}(8.2 \mathrm{kHz})$ and at $3 f_{n}$ $(24.6 \mathrm{kHz})$ in Fig. 7. The peak at $8.2 \mathrm{kHz}$ is $50 \%$ larger than the peak at $24.6 \mathrm{kHz}$ indicating that the peak at $8.2 \mathrm{kHz}$ is dominated by capacitive feedthrough. The experiment does not introduce any DC magnetic field with the electromagnet but there is an ambient magnetic field of $60 \mu \mathrm{T}$. The peak at $24.6 \mathrm{kHz}$ corresponds to an equivalent DC magnetic field of $2.4 \mathrm{mT}$ - the source of this peak is discussed below.

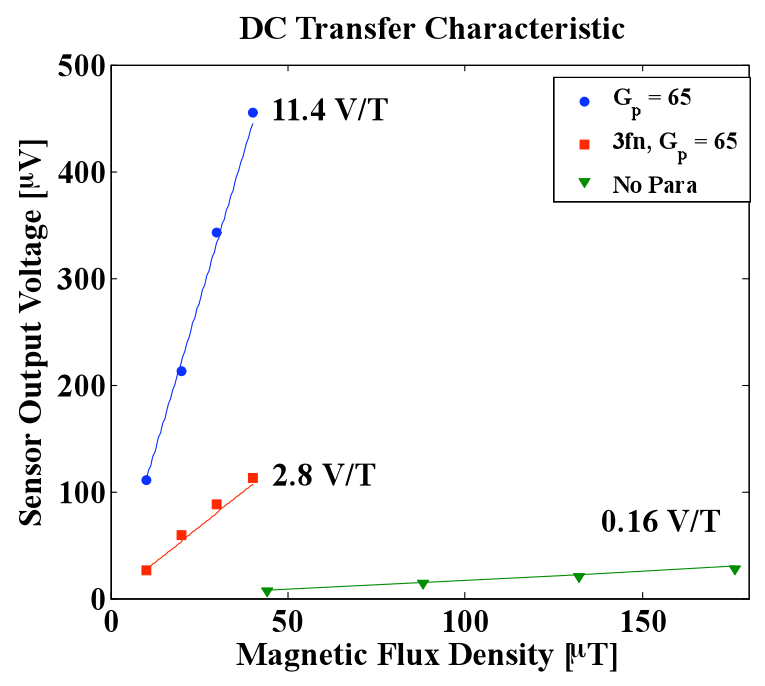

Figure 8. Transfer characteristic measured with and without parametric amplification demonstrating linear response.

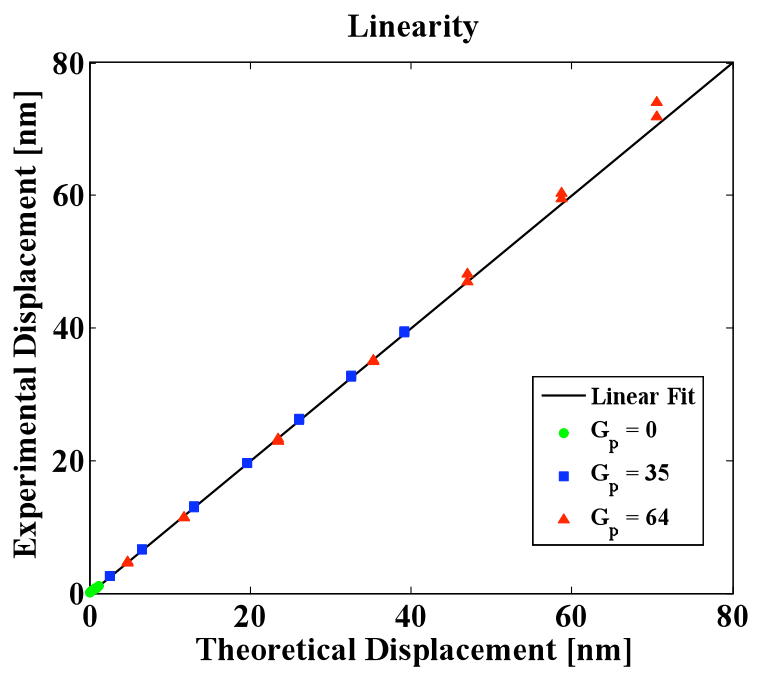

Figure 9. Linear theoretical versus experimental displacement for a $1 \mathrm{~Hz}$ input signal.

\section{Sensitivity}

The magnetic field transfer characteristic of the device is shown in Fig 8. The device is tested without parametric amplification, with 65 fold parametric amplification sensing at $f_{n}$ and 65 fold parametric amplification at $3 f_{n}$. If the device is not parametrically amplified the field sensitivity is $0.16 \mathrm{~V} / \mathrm{T}$. The field sensitivity of the device for a parametric gain of 65 is $11.4 \mathrm{~V} / \mathrm{T}$ and $2.8 \mathrm{~V} / \mathrm{T}$ when measured at $f_{n}$ and $3 f_{n}$, respectively.

\section{Sensor Linearity}

The dominant non-linearity in the device comes from the motion detection capacitors. This non-linearity is exploited to achieve parametric amplification but it also limits the sensor's output linearity. Since the onset of nonlinearity is determined by displacement, the maximum field input for linear response depends on the parametric gain. Fig. 9 shows the theoretical versus experimental displacement of the sensor for different parametric gains. For a maximum displacement of $46 \mathrm{~nm}$ and a parametric gain of 65 -fold the maximum linear field sensitivity is $68 \mu \mathrm{T}$. 


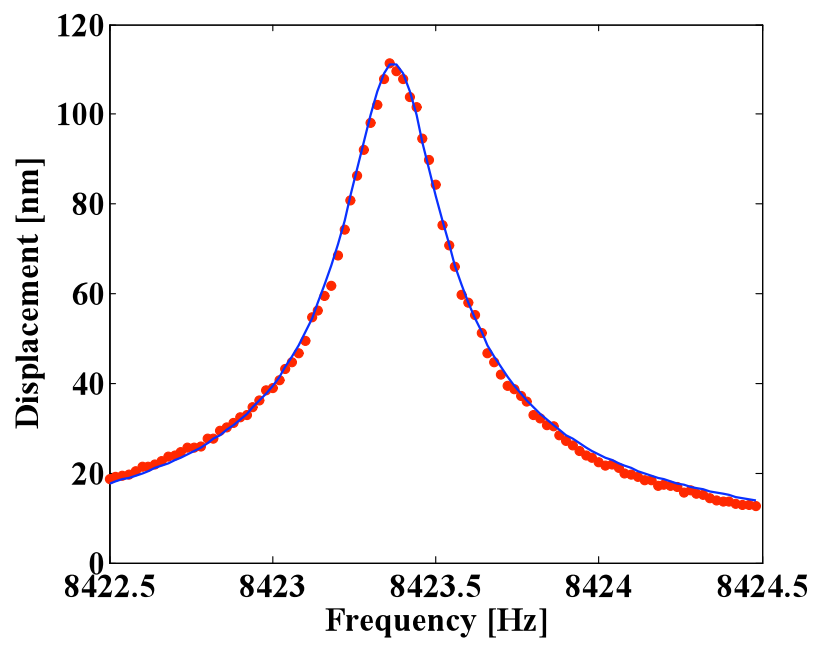

Figure 10. Magnetometer frequency response in a vacuum of 25 mTorr, a 97 mTesla DC magnetic field, drive current of $500 \mathrm{nA}$ and a capacitive bias of $2.5 \mathrm{~V}$.

\section{EXPERIMENTS IN A VACUUM}

An alternative method to increase the magnetometer's $Q$ is to operate the device in a 25 mTorr vacuum. Increasing $Q$ has a twofold benefit of lowering the Brownian noise floor and increasing the sensitivity. Most resonant MEMS devices are operated in a vacuum for the aforementioned reason.

\section{Frequency Response}

The sensor's frequency response is shown in Fig. 10 when measured in a DC magnetic field of $97 \mathrm{mT}$ by sweeping the frequency of the flexure current. The device was driven with a $500 \mathrm{nA}$ current and the capacitors were biased with $2.5 \mathrm{~V}$. The device has a natural frequency of $8423.4 \mathrm{~Hz}$ and a quality factor of 31,000 . If this device was operated with a $2.67 \mathrm{~mA}$ current then the device would have a Brownian noise limit of $3.4 \mathrm{nT} / \sqrt{\mathrm{Hz}}$.

\section{Brownian Noise measurement}

The Brownian noise spectrum is measured with a $5 \mathrm{~V}$ bias applied across the MEMS structure and zero flexure current, shown in Fig. 6, with a $14 \mathrm{pm} / \sqrt{ } \mathrm{Hz}$ equivalent electronic noise floor and a Brownian noise peak of $26 \mathrm{pm} / \sqrt{\mathrm{Hz}}$. The Brownian noise peak is equivalent to a resolution of $3.4 \mathrm{nT} / \sqrt{\mathrm{Hz}}$ when operated with a $2.67 \mathrm{~mA}$ flexure current which, in comparison with operation at atmospheric pressure, is a 25 -fold improvement over the parametrically amplified $(62 \mathrm{nT} / \sqrt{ } \mathrm{Hz})$ experiment and 295 -fold improvement over the electronically limited (1 $\mu \mathrm{T} / \sqrt{ } \mathrm{Hz})$ experiment.

\section{DISCUSSION}

The peak at $3 f_{n}(24.6 \mathrm{kHz})$ shown in Fig. 5 is not due to capacitive feedthrough or DC magnetic field. It is believed that a small residual $\mathrm{AC}$ voltage is present on the shuttle due to unbalanced flexure resistances resulting from small fabrication variations. The force created from the electrostatic actuation between the shuttle and the sense capacitors would also be parametrically amplified, corrupting the DC field measurement. To minimize this electrostatic force each flexure requires separate voltage adjustment to account for the mismatched resistances.

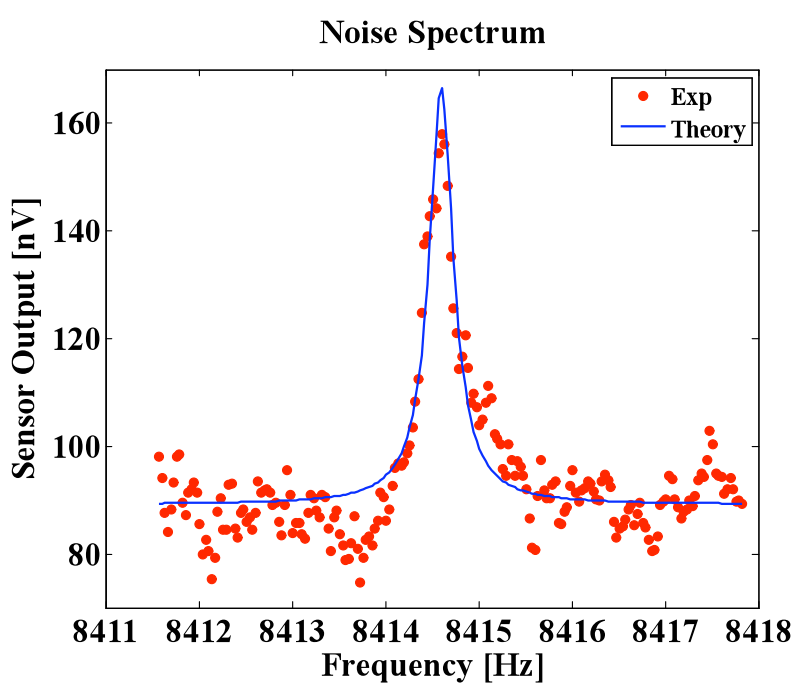

Figure 11. Noise measurement in a 25 mTorr vacuum with a $5 \mathrm{~V}$ capacitive bias.

\section{CONCLUSION}

Through this paper we have studied two methods for improving the $1 \mu \mathrm{T} / \sqrt{\mathrm{Hz}}$ resolution of a Lorentz force magnetometer.

Operating the device at atmospheric pressure, parametric amplification was performed which improves the performance in three ways. First, the force to displacement sensitivity was increased up to 85 fold. Secondly, the force sensitivity of the MEMS structure was increased until the sensor became Brownian noise limited, rather than electronic noise limited. Thirdly, the Lorentz force sensitivity was increased $40 \%$ more than the Brownian force sensitivity due to noise squeezing. For a drive current of $2.67 \mathrm{~mA}$ and a minimum parametric gain of 10 -fold the sensor has a noise resolution of $62 \mathrm{nT} / \sqrt{ } \mathrm{Hz}$.

Alternatively the device was operated in a $25 \mathrm{mTorr}$ vacuum where the device has a resolution of $3.4 \mathrm{nT} / \sqrt{\mathrm{Hz}}$ for a $2.67 \mathrm{~mA}$ drive current.

\section{ACKNOWLEDGEMENT}

This work was supported by NSF CAREER award ARRA CMMI0846379 .

\section{REFERENCES}

[1] H. Emmerich and M. Schofthaler, "Magnetic field measurements with a novel surface micromachined magneticfield sensor," IEEE Trans. Electron Dev., 47(5), pp. 972-977, (2000).

[2] M.J. Thompson and D.A. Horsley, "Resonant MEMS magnetometer with capacitive read-out," Proc. IEEE Sensors 2009, Christchurch, NZ, 992, (2009).

[3] M.J. Thompson and D.A. Horsley, "Parametrically-amplified MEMS magnetometer," Proc. 15th Int. Conf. Solid-State Sensors Actuators \& Microsystems (Transducers 2009), Denver, Colorado, 1194, (2009).

[4] D. Rugar and P. Grutter, "Mechanical parametric amplification and thermomechanical noise squeezing," Phys. Rev. Lett., 67(6), pp. 699-672, (1991).

\section{CONTACT}

*D.A. Horsley tel +1 530 752-1778; dahorsley@ucdavis.edu 